\title{
Orthostatic hypotension among elderly patients in Italian internal medicine wards: an observational study-comment
}

\author{
Hatice Bolek ${ }^{1}$ [ $\cdot$ Ertugrul Cagri Bolek ${ }^{2}$
}

Received: 26 June 2020 / Accepted: 8 July 2020 / Published online: 18 July 2020

(c) Società Italiana di Medicina Interna (SIMI) 2020

\section{Dear Editor,}

We recently read with great interest the article written by Pasina et al. with the title "Orthostatic hypotension among elderly patients in Italian internal medicine wards: an observational study", published in a past issue of "Internal and Emergency Medicine" [1]. Orthostatic hypotension affects $20 \%$ of the elderly population and it is associated with $35 \%$ increased risk in all caused mortality [2,3]. Although orthostatic hypotension is commonly seen and associated with poor outcomes, it can be overlooked in medical wards. So, the paper is worthy because it draws attention to high prevalence and importance of the orthostatic hypotension.

Although many causes of orthostatic hypotension were evaluated in the study, hemoglobin levels or anemia status of the patients were not discussed. Anemia is a common problem and it is associated with $80 \%$ increased mortality rate in hospitalized elderly patients [4]. Anemia may also cause or exacerbate the orthostatic hypotension and it is recommended to be evaluated in case of orthostatic hypotension. Anemia causes reduced systemic vascular resistance, which can result in orthostatic hypotension, by inducing nitric oxide synthesis and decreasing blood viscosity. On the other hand, the authors found that renal failure was a risk factor for persistent orthostatic hypotension in the study. Anemia is also very common in chronic renal failure patients and its incidence increases as glomerular filtration rate decreases. Liu et al. showed that reduced hemoglobin level causes impaired orthostatic blood pressure stabilization in renal failure and reduced hemoglobin can be one of main

Hatice Bolek

hati.kocc@gmail.com

1 Department of Internal Medicine, Hacettepe University Faculty of Medicine, Gevher Nesibe Street, Sihhiye, 06100 Ankara, Turkey

2 Division of Rheumatology, Department of Internal Medicine, Hacettepe University Faculty of Medicine, Ankara, Turkey contributors to orthostatic hypotension in patients with renal failure [5]. Anemia is not only associated with orthostatic hypotension and renal failure, but anemia is also associated with poor outcomes such as frailty, increased hospitalizations rates and increased mortality rates in elderly [4]. To sum up, anemia can be associated with orthostatic hypotension, renal failure and mortality which were evaluated in the study. Anemia and related factors may be examined in another analysis in the cohort of this comprehensive article can be fruitful.

Funding The authors have received no financial support or other benefits from commercial sources for the work reported here, and the authors have no other financial interests that could create a potential conflict of interest or the appearance of a conflict of interest regarding the present study.

\section{Compliance with ethical standards}

Conflict of interest The authors declare that they have no conflict of interest.

Human and animal rights statement This article does not contain any studies with human and participants or animal performed by any of the authors.

Informed consent For this type of study,formal consent is not required.

\section{References}

1. Pasina L, Casati M, Cortesi L, Tettamanti M, Pellegrini R, Oppedisano I et al (2020) Orthostatic hypotension among elderly patients in Italian internal medicine wards: an observational study. Intern Emerg Med 15(2):281-287

2. Angelousi A, Girerd N, Benetos A, Frimat L, Gautier S, Weryha $\mathrm{G}$ et al (2014) Association between orthostatic hypotension and cardiovascular risk, cerebrovascular risk, cognitive decline and falls as well as overall mortality: a systematic review and metaanalysis. J Hypertens 32(8):1562-1571 
3. Gupta V, Lipsitz LA (2007) Orthostatic hypotension in the elderly: diagnosis and treatment. Am J Med 120(10):841-847

4. Nathavitharana R, Murray J, d'Sousa N, Sheehan T, Frampton C, Baker B (2012) Anaemia is highly prevalent among unselected internal medicine inpatients and is associated with increased mortality, earlier readmission and more prolonged hospital stay: an observational retrospective cohort study. Intern Med J 42(6):683-691

5. Liu W, Wang L, Huang X, He W, Song Z, Yang J (2019) Impaired orthostatic blood pressure stabilization and reduced hemoglobin in chronic kidney disease. J Clin Hypertens 21(9):1317-1324
Publisher's Note Springer Nature remains neutral with regard to jurisdictional claims in published maps and institutional affiliations. 